\title{
Editorial \\ Current and Future Tests of General Relativity
}

\author{
Domenico Giulini ${ }^{1, *([)}$ and Philippe Jetzer ${ }^{2}$ (i) \\ 1 Institute for Theoretical Physics, Leibniz University of Hannover, 30167 Hannover, Germany \\ 2 Department of Physics, University of Zürich, 8057 Zürich, Switzerland; jetzer@physik.uzh.ch \\ * Correspondence: giulini@itp.uni-hannover.de
}

check for

updates

Citation: Giulini, D.; Jetzer, P.

Current and Future Tests of General

Relativity. Universe 2022, 8, 143.

https://doi.org/10.3390/universe

8030143

Received: 5 February 2022

Accepted: 16 February 2022

Published: 23 February 2022

Publisher's Note: MDPI stays neutral with regard to jurisdictional claims in published maps and institutional affiliations.

Copyright: (C) 2022 by the authors. Licensee MDPI, Basel, Switzerland This article is an open access article distributed under the terms and conditions of the Creative Commons Attribution (CC BY) license (https:// creativecommons.org/licenses/by/ $4.0 /)$.
General Relativity (GR) holds a special place amongst all fundamental theories of physics: on one hand, it is the theory of all gravitational phenomena; on the other hand, it is also a theory of spacetime. However, the structure of spacetime enters all other physical theories of interactions at the most fundamental level. Hence, as a matter of principle, the status of GR cannot be thought of as isolated from the rest of fundamental physics. According to GR, gravity is not just one amongst four independently rooted interactions. If, under certain physical conditions, GR turns out to fail, this will inevitably also effect the rest of physics. Testing GR up to the most extreme conditions imaginable is therefore not just an end in itself, it also means probing the very foundations of physics. For further discussion on the present and future perspectives on gravitation and cosmology, see the Special Issue celebrating the centennial of GR in this journal, in particular [1,2] and references therein.

In this Special Issue we did not attempt to collect a representative cross-section of all ongoing research regarding all tests of GR. This would indeed have been a formidable task, and at the completion of which we would probably have to revise its beginning due to the already very fast and still accelerating developments in observational and experimental techniques. In addition, there already exist impressive collections and discussions covering most of the research up to about ten years ago, such as [3]. Rather, the task we set ourselves was to look at certain typical questions via selected papers by outstanding contributors. This may be looked upon as regarding cutting-edge research through a magnifying glass.

Our collection of six papers contains three reviews on a broader scale and three contributions that focus on more particular issues. In the order of submission they are:

1. "Revisiting the 2PN Pericenter Precession in View of Possible Future Measurements" by Lorenzo Iorio (19 pages);

2. "Gravity Tests with Radio Pulsars" by Norbert Wex and Michael Kramer (38 pages);

3. "Benefit of New High-Precision LLR Data for the Determination of Relativistic Parameters" by Liliane Biskupek, Jürgen Müller and Jean-Marie Torre (13 pages);

4. "On the 2PN Pericentre Precession in the General Theory of Relativity and the Recently Discovered Fast-Orbiting S-Stars in Sgr A*" by Lorenzo Iorio (12 pages);

5. "On the 2PN Periastron Precession of the Double Pulsar PSR J0737-3039A/B" by Lorenzo Iorio (12 pages);

6. "Testing General Relativity with Gravitational Waves: An Overview" by Naderi Varium Krishnendu and Frank Ohme (21 pages).

Research into radio pulsars has been, and will be for a long time to come, one of the most fruitful, most rich, and also most complex part of physics, involving essentially all of its disciplines [4]. Much new physics, not only gravitational, can be expected from pursuing this line of research, but the computational efforts required are enormous.

Gravity-wave research has recently undergone a dramatic transformation process from mere detection into a powerful observational tool and thereby one of the main supporting pillars in a new era of multimessenger astronomy [5]. It currently dominates observational gravity research worldwide and will almost certainly lead to many unexpected new insights 
into early cosmology as well as the physics of compact objects, in particular black holes and neutron stars.

Despite the spectacular aspects of the areas mentioned, which are often exclusively mentioned in public talks and also as "selling points" for gravity research, one should not forget that solar system and near-Earth gravity research is by no means closed, and that GR is also becoming a tool for studying the Earth's environment. Relativistic Geodesy and inertial sensing are closely linked to GR and rely on its predictions, and can, conversely, be used for high-precision tests of GR [6]. Present and future perspectives for further solar-system tests are discussed in [7].

However, last but not least, it needs to be mentioned that eventually everything depends on getting the details right. The "grand picture", beautiful as it may appear from a distance, should not be trusted unless all details have been checked independently. Calculations based on post-Newtonian approximation schemes are central but delicate and need to be cross-checked independently [8-10]. How much goes into this, and how much can be drawn from it, can be nicely exemplified by the pericenter precession that more than 100 years ago led to the first stunning success of GR.

Conflicts of Interest: The authors declare no conflict of interest.

\section{References}

1. Iorio, L. Editorial for the Special Issue 100 Years of Chronogeometrodynamics: The Status of the Einstein's Theory of Gravitation in Its Centennial Year. Universe 2015, 1, 38-81. [CrossRef]

2. Debono, I.; Smoot, G.F. General Relativity and Cosmology: Unsolved Questions and Future Directions. Universe 2016, 2, 23. [CrossRef]

3. Will, C. The Confrontation between General Relativity and Experiment. Living Rev. Relativ. 2014, 17, 4. [CrossRef] [PubMed]

4. Wex, N.; Kramer, M. Gravity Tests with Radio Pulsars. Universe 2020, 6, 156. [CrossRef]

5. Krishnendu, N.V.; Ohme, F. Testing General Relativity with Gravitational Waves: An Overview. Universe 2021, 7, 497. [CrossRef]

6. Biskupek, L.; Müller, J.; Torre, J.M. Benefit of New High-Precision LLR Data for the Determination of Relativistic Parameters. Universe 2021, 7, 34. [CrossRef]

7. De Marchi, F.; Cascioli, G. Testing general relativity in the solar system: present and future perspectives. Class. Quantum Gravity 2020, 37, 095007. [CrossRef]

8. Iorio, L. On the 2PN Periastron Precession of the Double Pulsar PSR J0737-3039A/B. Universe 2021, 7, 443. [CrossRef]

9. Iorio, L. On the 2PN Pericentre Precession in the General Theory of Relativity and the Recently Discovered Fast-Orbiting S-Stars in Sgr $A^{*}$. Universe 2021, 7, 37. [CrossRef]

10. Iorio, L. Revisiting the 2PN Pericenter Precession in View of Possible Future Measurements. Universe 2020, 6, 53. [CrossRef] 